\title{
Uso de anticoagulación al alta hospitalaria en pacientes con insuficiencia cardíaca y fibrilación auricular
}

\author{
Use of Anticoagulation at the Time of Discharge in Patients With Heart Failure and \\ Atrial Fibrillation
}

\author{
Ignacio Mosquera Pérez, Javier Muñiz García, Evaristo Freire Castroseiros, Alberto \\ García Castelo y Alfonso Castro-Beiras, por el grupo de trabajo del estudio \\ INCARGAL
}

\begin{abstract}
Resumen
Introducción y objetivos. Evaluar el grado de cumplimiento de las guías sobre uso de anticoagulación crónica en pacientes con insuficiencia cardíaca y fibrilación auricular.

Pacientes y método. Se usó la base de datos del estudio INCARGAL, analizando datos de 195 pacientes consecutivos (88 varones, edad $76 \pm 10$ años) admitidos con ambos diagnósticos en tres hospitales gallegos entre enero y junio de 1999. Se asumió que todos deberían de haber recibido anticoagulación al alta hospitalaria en ausencia de contraindicaciones. Se comparó el tratamiento al alta (anticoagulación o no) con la presencia o ausencia de contraindicaciones.

Resultados. Un total de 152 pacientes (78\%) no tenían contraindicaciones para la anticoagulación y 43 presentaban alguna (absoluta, 11; relativa, 32). De los pacientes sin contraindicación, sólo recibieron anticoagulación al alta el $50 \%$. Ningún paciente con contraindicación absoluta y tres con contraindicación relativa recibieron anticoagulación. La prescripción de anticoagulación en los pacientes sin contraindicaciones fue menor en los que tenían una mayor edad, antecedente de cardiopatía isquémica, ausencia de valvulopatía, uso de bloqueadores beta, no realización de ecocardiograma e ingreso en un servicio diferente del de cardiología ( $\mathrm{p}<0,05)$. En el análisis multivariante, la edad, el infarto de miocardio previo y la ausencia de valvulopatía significativa permanecieron como predictores independientes de menor uso de anticoagulación.

Conclusiones. El empleo de anticoagulación al alta hospitalaria en pacientes sin contraindicación para su uso, con fibrilación auricular e insuficiencia cardíaca, es menor del recomendado. La edad avanzada disminuye su empleo. La presencia de otras indicaciones para la antiagregación o la anticoagulación parecen determinar la elección de una u otra terapia. No hubo mala adecuación por exceso de prescripción.
\end{abstract}

\footnotetext{
Abstract

Introduction and objectives. To assess the degree of compliance with current guidelines for chronic anticoagulation in patients with heart failure and atrial fibrillation.

Patients and method. From the INCARGAL Study database, we analyzed data from 195 consecutive patients (88 men; mean age $76 \pm 10$ years) with both conditions, admitted to three Galician hospitals between January and March 1999. It was assumed that these patients should have received anticoagulant therapy at discharge, unless contraindicated. We studied the association of treatment at discharge (anticoagulation or not) with the presence or absence of contraindications.

Results. - 152 patients (78\%) had no contraindication for anticoagulation and 43 had at least one (absolute: 11, relative: 32 ). Only $50 \%$ of patients without contraindications received anticoagulation at the time of discharge. No patient with an absolute contraindication and 3 with a relative one received anticoagulation. Factors related with the less frequent prescription of anticoagulation therapy in patients without a formal contraindication were: age, a previous history of coronary heart disease, absence of valvular heart disease, prior' myocardial infarction, treatment with beta-blocking agents, non performance of an echocardiogram, and admission to a department other than cardiology. On multivariate analysis, age, prior myocardial infarction, and non-valvular disease were found to be independent predictors of less use of anticoagulation

Conclusions. Anticoagulant therapy is used less often than recommended at discharge in patients with heart failure and atrial fibrillation for whom there were no contraindications. Advanced age reduces its use. The presence of other indications for antiplatelet or anticoagulation therapy appears to determine the choice of one or the other. Noncompliance with the guidelines due to overprescription was not found.
} 
Palabras clave: Anticoagulantes. Fibrilación auricular. Insuficiencia cardíaca.

Key words: Anticoagulants. Atrial fibril/ation. Heart failure.

Abreviaturas: FA: fibrilación auricular. IC: insuficiencia cardíaca. HBPM: heparinas de bajo peso molecular. ACV: accidente cerebrovascular. lECA: inhibidor de la enzima de conversión de la angiotensina. ARA-U: antagonista de los receptores de la angiotensina II

\section{Introducción}

La fibrilación auricular (FA) y la insuficiencia cardíaca (IC) afectan, cada una de ellas, al 1-2\% de la población general y la prevalencia de ambas aumenta rápidamente con la edad ${ }^{1,2}$. Comparten factores de riesgo común y coexisten frecuentemente, de manera que la IC puede afectar a más del $50 \%$ de los pacientes con FA3, mientras que la prevalencia de $\mathrm{FA}^{3}$ se incrementa en proporción a la gravedad de la IC crónica ( $10 \%$ en NYHA II-III ${ }^{4}, 48 \%$ en NYHA IV ${ }^{5}$ ).

La FA no reumática se asocia a una tasa de accidentes cerebrovasculares (ACY) cinco veces superior a la de los pacientes sin $\mathrm{FA}^{6}$ la tasa de ACY varía entre el 1-8\% anual según la edad y la presencia o no de otros factores de riesgo embolígeno ${ }^{7}$. Uno de estos factores de riesgo embolígeno es la presencia de IC o de disfunción ventricular, que multiplica por tres la tasa de $\mathrm{ACV}^{8}$. Debido a ello, la disminución en la incidencia de ACY mediante la anticoagulación crónica en pacientes con FA no reumática observada en diversos estudios $^{7-17}$ es especialmente importante en el subgrupo de pacientes con le. Las guías de la Sociedad Española de Cardiología sobre práctica clínica en arritmias ${ }^{18}$ y sobre el uso del tratamiento antitrombótico en cardiología ${ }^{19}$, y las guías conjuntas del American College of Cardiology/American Heart Association/European Society of Cardiology sobre el tratamiento de la $\mathrm{FA}^{20}$ consideran como indicación de clase I el empleo de anticoagulación oral crónica en todos los pacientes con IC y FA, salvo que existan contraindicaciones para dicha terapia, en cuyo caso se recomienda el empleo de aspirina a dosis de $325 \mathrm{mg} /$ día.

El grado de cumplimiento de estas recomendaciones es desconocido. Estudios realizados sobre poblaciones con FA no reumática en general (no específicamente en aquellos con IC) en España ${ }^{21,22}$ indican una utilización de anticoagulantes en pacientes de alto riesgo embolígeno inferior al $50 \%$. Un estudio reciente realizado en Suecia $^{23}$ mostró que, aunque el $94 \%$ de los médicos encuestados consideraban que los pacientes con FA y factores de riesgo embolígeno deberían recibir anticoagulación crónica, sólo el $40 \%$ de estos pacientes pertenecientes a un área sanitaria concreta estaban recibiendo warfarina. En general, todos estos estudios coinciden en señalar que existe un amplio margen de mejora en la utilización de estos fármacos, pero no tienen en cuenta las posibles contraindicaciones para dicho tratamiento.

Conocida la escasa frecuencia de utilización del tratamiento anticoagulante en la FA, el objeto de este estudio es analizar la adecuación de la prescripción de fármacos anticoagulantes en pacientes con FA no reumática e IC en Galicia, teniendo en cuenta la presencia o ausencia de contraindicaciones para la misma, y explorar los factores relacionados con dicha adecuación. Hasta donde sabemos, no existe ningún artículo que aborde este aspecto concreto, pregunta que analiza el presente trabajo.

\section{Pacientes y método}

\section{Pacientes}

Se utilizó la base de datos del Registro de Insuficiencia Cardíaca en Galicia (INCARGAL), descrito previamente ${ }^{24}$ De manera resumida, se trata de un registro hospitalario en el que se recogieron de manera transversal, siguiendo un protocolo previamente especificado, datos de los ingresos consecutivos por IC realizados por los servicios participantes de 14 hospitales gallegos entre los meses de enero y junio de 1999. Al final del período de inclusión, el registro contenía datos de 951 ingresos correspondientes a 837 pacientes, de los que 435 (52\%) padecían FA crónica o paroxística. Con el objeto de poder estimar la adecuación se hizo necesario disponer de información sobre posibles contraindicaciones para el empleo de anticoagulación crónica; dado que estas contraindicaciones no se contemplaban en el cuaderno de recogida de datos del INCARGAL, se revisaron nuevamente las historias clínicas de todos los pacientes incluidos en este trabajo. Por motivos de conveniencia, se redujo la muestra a tres de los hospitales, que habían participado en el registro (Complejo Hospitalario Juan Canalejo de A Coruña, Complejo Hospitalario de Lugo y Complejo Hospitalario de Ourense), el primero con unidad de hemodinámica y 
servicio de cirugía cardíaca y los otros dos con servicio de cardiología y docencia general pero no específica de cardiología. La muestra final está compuesta por 214 pacientes con FA (49\% del total de pacientes en FA del registro original); no existían diferencias estadísticamente significativas entre estos pacientes y los del conjunto de hospitales del registro INCARGAL global (datos no expuestos).

\section{Definiciones}

De acuerdo con las guías actuales sobre el tratamiento de la FA, se consideró que todos estos pacientes tenían indicación de clase I para recibir anticoagulación crónica, siempre que no existieran contraindicaciones para su uso. Se consideraron contraindicaciones para el uso de anticoagulación crónica (contraindicación en lo sucesivo) cualquiera de las recogidas en las Guías de la Sociedad Española de Cardiología sobre el uso de terapia antitrombótica en cardiología (tabla 1). No se incluyó la edad avanzada como contraindicación relativa ya que ninguna de las guías actuales sobre FA la recoge como tal y simplemente se especifica que los pacientes mayores deben de seguir controles más frecuentes y regímenes de anticoagulación menos exigentes.

Tabla 1. Contraindicaciones para uso de anticoagulación crónica (según las guías de la Sociedad Española de Cardiología sobre el uso de terapia antitrombótica en cardiología)

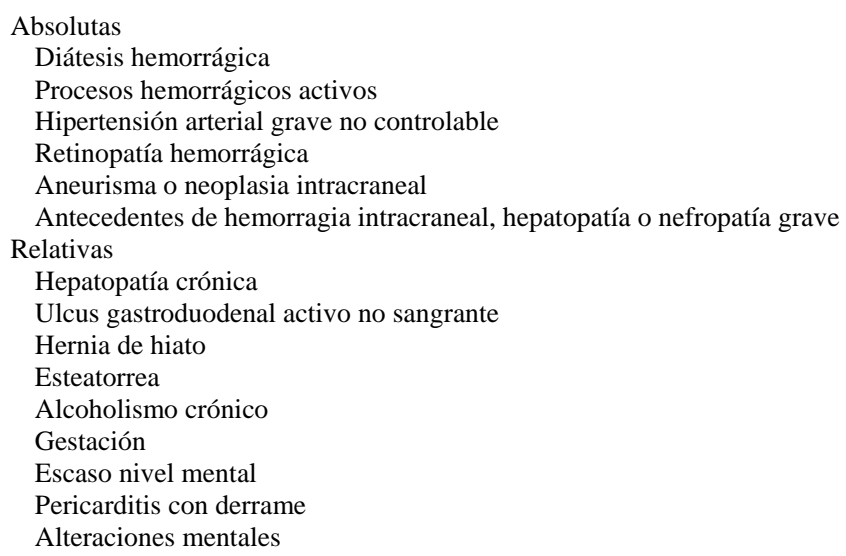

Se consideró que un paciente estaba anticoagulado si en el momento del alta estaba recibiendo anticoagulación oral y/o heparina de bajo peso molecular (HBPM), En los pacientes sin contraindicaciones se definió el correcto seguimiento de las guías como la prescripción de anticoagulación al alta hospitalaria, considerándose su ausencia (aun en pacientes con tratamiento antiagregante) un mal cumplimiento de ellas.

\section{Variables recogidas}

Las variables relacionadas directamente con los objetivos del estudio fueron la presencia o ausencia de contraindicaciones y la prescripción o no al alta de tratamiento anticoagulante. Además, se recogieron las siguientes variables: edad, sexo, lugar de residencia (urbano o rural), hospital, tipo de servicio (cardiología u otros), tipo de cardiopatía, historia de hipertensión arterial, antecedente de ACV previo, fracción de eyección, tratamiento al alta con otros fármacos (antiagregantes, bloqueadores beta, IECA, ARA-U) y realización de pruebas di agnósticas (ecocardiograma, cateterismo cardíaco). Estos fármacos y pruebas se escogieron como posibles marcadores del grado de cumplimiento de otras recomendaciones en pacientes con $1 \mathrm{C}$ o de la dotación del hospital en cuestión. 


\section{Análisis estadístico}

Los datos cuantitativos se expresan como media \pm desviación estándar y los cualitativos como número absoluto (porcentaje). Se comparó el tratamiento recibido (anticoagulación o no) con la presencia o ausencia de contraindicaciones. En los pacientes sin contraindicaciones, se realizó un análisis univariado mediante el test de la $\chi^{2}$ para valorar si alguna variable se relacionaba con el correcto seguimiento de las guías. Posteriormente, se realizó análisis de regresión logística considerando como variable dependiente el uso o no de anticoagulación y como variables independientes las que habían mostrado una asociación estadísticamente significativa en el modelo univariado. En los resultados sólo se presentan las que permanecieron finalmente en el modelo.

Se consideraron resultados estadísticamente significativos aquellos con valores de $\mathrm{p}<0,05$. Todos los cálculos se llevaron a cabo mediante el paquete estadístico SPSS para Windows, versión 10.0.

\section{Resultados}

De los 214 pacientes, 201 fueron dados de alta vivos. De seis de estos pacientes no se disponía de datos sobre posibles contraindicaciones, por lo que los análisis posteriores se efectuaron con los 195 pacientes restantes (88 varones y 107 mujeres; edad $76 \pm 10$ años; rango, 44-92). En 95 pacientes (49\%) éste era su primer ingreso, mientras que $100(51 \%)$ habían tenido al menos un ingreso previo por IC (mediana, 2; rango, 1-10). Se disponía de datos sobre la función sistólica de 119 pacientes (61\%): la fracción de eyección era superior al $50 \%$ en el $60 \%$ de éstos, del 35-50\% en el $23 \%$ e inferior a $35 \%$ en el $17 \%$.

Un total de 43 pacientes $(22,1 \%$ ) presentaban alguna contraindicación (tabla 2): absoluta en 11 $(5,7 \%)$ y relativa en $32(16,4 \%)$. Ninguno de los pacientes con contraindicación absoluta recibió tratamiento anticoagulante, y sólo tres con contraindicación relativa lo recibieron (uno con hernia de hiato, uno con alteraciones mentales y uno con alcoholismo crónico).

Tabla 2. Tratamiento antitrombótico al alta en función de la presencia de contraindicaciones para anticoagulación

\begin{tabular}{lcccc}
\hline & $\begin{array}{c}\text { Sin contraindicación } \\
(\mathrm{n}=152)\end{array}$ & $\begin{array}{c}\text { Contraindicación relativa } \\
(\mathrm{n}=32)\end{array}$ & $\begin{array}{c}\text { Contraindicación absoluta } \\
(\mathrm{n}=11)\end{array}$ & $\begin{array}{c}\text { Total } \\
(\mathrm{n}=195)\end{array}$ \\
\hline Ninguno & $20(13 \%)$ & $11(35 \%)$ & $7(64 \%)$ & $38(19,5 \%)$ \\
Antiagregación & $56(37 \%)$ & $18(56 \%)$ & $4(36 \%)$ & $78(40 \%)$ \\
Anticoagulación & $76(50 \%)$ & $3(9 \%)$ & $\mathrm{O}$ & $79(40,5)$ \\
\end{tabular}

Entre paréntesis se expresa e[ porcentaje que representa de cada columna.

El 77,9\% de los pacientes no tenían ninguna contraindicación, De éstos, 79 (50\%) recibieron anticoagulación al alta (71 sólo anticoagulación oral, cuatro exclusivamente HBPM y cuatro ambos tratamientos). El análisis univariado dentro del grupo sin contraindicaciones mostró que las variables relacionadas con un menor seguimiento de las guías eran (tabla 3): mayor edad, antecedentes de cardiopatía isquémica o infarto de miocardio previo, ausencia de valvulopatía, ausencia de tratamiento con bloqueadores beta, ausencia de realización de ecocardiograma e ingreso en otro servicio diferente del de cardiología. 
Tabla 3. Variables demográficas y clínicas de los pacientes sin contraindicaciones para anticoagulación en función de si se siguieron o no las guías al alta hospitalaria

\begin{tabular}{|c|c|c|c|c|}
\hline & Anticoagulación & Antiagregación o nada & $\mathrm{p}$ & Total \\
\hline Edad :2: 75 años & 34,2 & 77,0 & $<0,001$ & 55,3 \\
\hline Sexo varón & 48,7 & 43,4 & 0,515 & 46,1 \\
\hline Hospital & & & 0,840 & \\
\hline Servicio de cardiología & 51,3 & 31,6 & 0,014 & 41,4 \\
\hline Residencia rural & 56 & 62,2 & 0,445 & 59,1 \\
\hline Ingresos previos por insuficiencia cardíaca & 53,9 & 47,4 & 0,422 & 50,7 \\
\hline Cardiopatía isquémica & 14,5 & 28,9 & 0,033 & 21,7 \\
\hline Infarto de miocardio previo & 2,6 & 14,5 & 0,009 & 8,6 \\
\hline Valvulopatía significativa & 42,1 & 21,1 & 0,006 & 31,6 \\
\hline HTA & 51,3 & 65,8 & 0,071 & 58,6 \\
\hline Diabetes & 18,7 & 24 & 0,430 & 21,3 \\
\hline Hipercolesterolemia & 17,1 & 17,1 & 1,000 & 17,1 \\
\hline Historia de tabaquismo & 30,2 & 25 & 0,582 & 29,2 \\
\hline EPDC & 28,9 & 36,8 & 0,301 & 32,9 \\
\hline ACV previo & 15,8 & 11,8 & 0,482 & 13,8 \\
\hline Arteriopatía periférica & 7,9 & 10,5 & 0,578 & 9,2 \\
\hline Fracción de eyección $<50 \%$ & 46,6 & 28,9 & 0,087 & 39,6 \\
\hline Bloqueadores beta & 13,2 & 2,6 & 0,016 & 7,9 \\
\hline IECA & 63,2 & 65,8 & 0,741 & 64,5 \\
\hline Ecocardiog rafía & 72,4 & 44,7 & 0,001 & 58,6 \\
\hline Cateterismo & 3,9 & 11,8 & 0,085 & 7,9 \\
\hline
\end{tabular}

Todos [os valores expresan el porcentaje. En hospital no se indican porcentajes por ser una variable con más de dos categorias. ACV: accidente cerebrovascular: EPOC: enfermedad pulrnonar obstructiva crónica; HTA: hipertensión arterial; [ECA: inhibidor de [a enzima de conversión de [a an-

giotensina.

Todas estas variables se incluyeron en un análisis multivariado, en el que se comprobó que sólo la edad, el antecedente de infarto de miocardio y la ausencia de valvulopatía significativa se relacionaban de manera independiente e inversa con el conecto seguimiento de las guías (tabla 4).

Tabla 4. Factores relacionados con el uso de anticoagulación al alta en pacientes sin contraindicación para la misma, según el análisis de regresión logística múltiple

\begin{tabular}{lccc}
\hline & Odds ratio & IC del 95\% & $\mathrm{p}$ \\
\hline Edad $\geq 75$ años & 0,88 & $0,83-0,92$ & $<0,001$ \\
Infarto de miocardio previo & 0,16 & $0,03-0,77$ & 0,023 \\
Valvulopatía & 2,89 & $1,25-6,69$ & 0,013 \\
& & \\
\hline
\end{tabular}




\section{Discusión}

El empleo de anticoagulación oral crónica en los pacientes con FA no reumática y algún factor de riesgo tromboembólico asociado ha demostrado una reducción relativa del riesgo de ACV y embolia periférica del $64 \%$ anual, con una disminución absoluta de riesgo del 3,] \%, sin un aumento significativo de la tasa de hemorragias mayores con respecto al uso de aspirina cuando se mantiene un INR de $2-3^{7,8.25-}$ ${ }^{29}$. Debido a ello, las principales guías sobre el tratamiento de la FA consideran la administración de anticoagulantes orales a pacientes con FA e IC como indicación de clase I. Una utilización menor de la recomendable, sin que exista ninguna contraindicación, supone la pérdida de dichos beneficios, en el sentido sobre todo de un mayor número de ACV isquémicos (de los cuales el 50-70\% conducen a la muerte o a déficit neurológicos graves ${ }^{30}$ ).

El principal hallazgo de nuestro trabajo es que las tres cuartas partes de los pacientes no presentaban ninguna contraindicación y, sin embargo, solamente la mitad de éstos se fueron de alta hospitalaria recibiendo anticoagulación. En estos pacientes sin contraindicaciones parece existir una relación inversa e independiente entre el correcto seguimiento .de las guías y tres variables: la edad, el antecedente de infarto de miocardio y la ausencia de valvulopatía significativa.

La relación inversa con la edad (fig. 1) probablemente tiene que ver con la preocupación por el aumento del riesgo hemorrágico en personas mayores. Sin embargo, se sabe que en pacientes mayores de 75 años con algún factor de riesgo embolígeno asociado, la anticoagulación disminuye el riesgo de ACV con respecto al placebo en un $73 \%$ y con respecto a aspirina en un $40 \%$, a costa de un ligero aumento del riesgo de sangrado mayor que no anula el beneficio observado ${ }^{7}$. El único ensayo clínico importante que ha detectado en pacientes de edad avanzada un exceso de sangrados suficiente como para anular el efecto beneficioso de la anticoagulación ha sido el SPAF-II ${ }^{15}$, en el que en mayores de 75 años hubo unas tasas anuales de hemorragia mayor del 4,2\% y de hemorragia intracraneal del 1,8\%. Los propios autores del estudio observaron, en un análisis posterior ${ }^{28}$, que el principal factor relacionado con el riesgo de hemorragia había sido la intensidad de la anticoagulación. Por ello, las guías sobre la FA sólo especifican que, en pacientes mayores de 75 años, se debe seguir controles de anticoagulación más frecuentes y regímenes menos agresivos, buscando un INR cercano a 2.

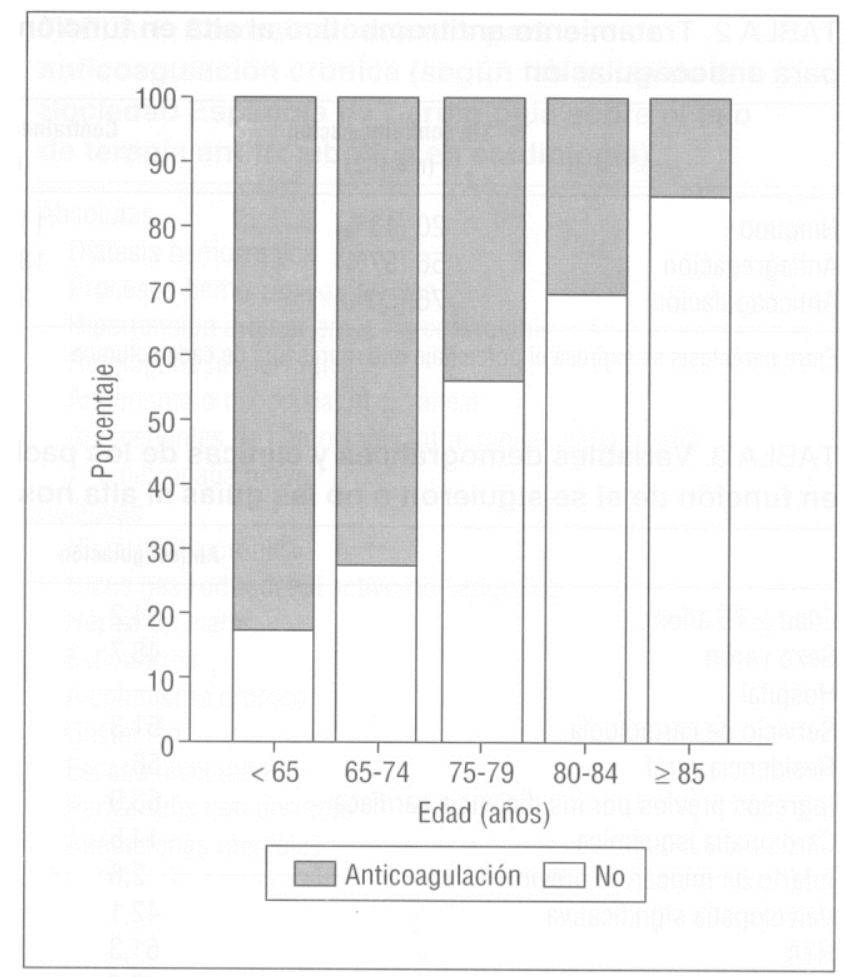

Fig. 1. Relación entre edad y uso de anticoagulación en los pacientes sin contraindicación para la misma. 
No se ha aclarado si el beneficio de la anticoagulación supera el riesgo hemorrágico en los pacientes muy ancianos. Conviene recordar que los estudios más amplios han incluido a una población relativamente joven, por lo que sus conclusiones pueden no ser aplicables a grupos de edad muy avanzada. Fihn et $\mathrm{al}^{27}$, en un estudio que incluía a 93 pacientes mayores de 80 años, observaron en ellos una tasa anual de hemorragia seria y amenazante y/o fatal del 4,4 y del 3,4\%, respectivamente. Incluso en este grupo de gente mayor, el principal factor de riesgo hemorrágico fue la intensidad de la anticoagulación, por lo que concluyeron que posiblemente no esté justificado negar el tratamiento anticoagulante simplemente según la edad.

El segundo factor relacionado con una menor prescripción de anticoagulación al alta es el antecedente de infarto de miocardio previo. Esto posiblemente esté relacionado con el uso habitual de antiagregantes en pacientes con cardiopatía isquémica crónica. Si bien la aspirina ha demostrado una reducción del número de episodios después de un infarto de miocardio con respecto a placebo en torno al 10-20\%, la anticoagulación oral crónica reduce los episodios en la misma proporción ${ }^{31,32}$; además, los pocos estudios comparativos directos entre aspirina y anticoagulación oral no han detectado diferencias entre ambas, tanto en la era precomo postrornbolítica ${ }^{33,34}$. Debido a que la aspirina es barata y no necesita monitorización, es la terapia antitrombótica más usada actualmente después de un infarto de miocardio; sin embargo, en presencia de otra indicación de tratamiento anticoagulante (como ocurre en nuestros pacientes), este último parece el tratamiento preferible ${ }^{35,36}$.

La relación positiva entre anticoagulación al alta hospitalaria y la presencia de valvulopatía significativa probablemente refleja el efecto derivado de la acumulación de indicaciones para anticoagulación en un paciente concreto, ya que la valvulopatía mitral y/o aórtica significativa por sí sola es un criterio de anticoagulación en pacientes con FA.

Ningún paciente fue dado de alta con los dos tipos de tratamiento (anticoagulación y antiagregación) $\mathrm{y}$, de los pacientes sin contraindicaciones, un porcentaje similar se fue con una u otra terapia. Esto probablemente refleja dos aspectos: se tiende a evitar la asociación de estos fármacos y, en muchos casos, todavía se prefiere antiagregar que anticoagular. En cuanto a la asociación, hoy día no está claro si con ella se consigue un mayor beneficio o sólo conduce a un riesgo de hemorragia inaceptable; las guías únicamente la recomiendan cuando, a pesar de una correcta anticoagulación, se produce un episodio embólico. Sin embargo, el segundo aspecto sí parece reflejar un mal cumplimiento de las recomendaciones ya que, si bien la aspirina es superior al placebo para prevenir tromboembolismos, su uso en un paciente que es candidato a tratamiento anticoagulante supone una merma del beneficio potencialmente alcanzable. Aunque se acepta que la antiagregación tiene un menor riesgo de hemorragia (sobre todo de hemorragia menor), en el estudio AFASAK- $2^{37}$ se observó una tasa de hemorragias mayores con aspirina ligeramente superior que con warfarina (1,4 frente al, $1 \%$ anual); si bien la diferencia no era estadísticamente significativa, los autores resaltaban que la aspirina no es un tratamiento tan inocuo como tantas veces se cree.

No se han encontrado diferencias en el uso de anticoagulación en relación con el sexo, lugar de residencia, hospital, tipo de servicio médico, pruebas diagnósticas realizadas o terapia concomitante al alta hospitalaria (con la excepción de los antiagregantes).

De los 43 pacientes con alguna contraindicación, sólo tres con alguna contraindicación relativa fueron dados de alta con anticoagulación, lo cual sugiere que sus médicos estuvieron muy pendientes de la presencia de posibles contraindicaciones y que no hay problemas por un «exceso» de tratamiento. De los restantes 40 pacientes con contraindicaciones, únicamente el $45 \%$ fueron dados de alta con algún antiagregante. La baja proporción de empleo de antiagregación en este subgrupo podría estar motivada por: a) algunas de las contraindicaciones para anticoagular a un paciente lo son también para antiagregarlo, y b) la edad avanzada de los pacientes con contraindicaciones (mediana, 83,3 años; rango, 57-99 años) y la comorbilidad importante que reflejan algunas de ellas pueden desanimar al médico a adoptar medidas de prevención «no imprescindibles».

\section{Limitaciones}

Se trata de un estudio transversal, por lo que escapa del alcance del mismo el conocer el efecto del tratamiento anticoagulante sobre la morbimortalidad futura.

No se incluyó a todos los pacientes del registro original, solamente a los que ingresaron en tres hospitales concretos. Este aspecto, que de todos modos no afecta a la validez interna de los datos, parece poco relevante en cuanto a la posibilidad de generalización de los mismos, ya que dichos hospitales son los que más pacientes habían recogido y no se detectaron diferencias entre estos pacientes y el global del registro original.

El registro INCARGAL no recogió el valor de INR en los pacientes con tratamiento anticoagulante, por lo que no es posible conocer el número de pacientes que recibieron correctamente este tratamiento. 
Tampoco recogía la presencia o ausencia de cirugía de sustitución valvular con prótesis mecánicas, dato de gran importancia a la hora de decidir la necesidad de anticoagulación.

Al ser un estudio transversal, sólo se pudo analizar el tratamiento en el momento del alta. Es posible que durante el seguimiento realizado en consultas externas, sobre todo en servicios que dispongan de la posibilidad de una revisión temprana tras el alta, varíen las frecuencias de uso de estos fármacos.

\section{Conclusiones}

El empleo de tratamiento anticoagulante al alta hospitalaria en pacientes con IC y FA es menor del recomendado por las principales guías actualmente en vigor, sobre todo en los pacientes de edad avanzada. En muchos casos, todavía se prefiere optar por una actitud intermedia, como el empleo de antiagregantes, con la consiguiente pérdida de beneficio potencial. Variables tales como el medio de residencia del paciente, hospital de ingreso o tipo de servicio médico no parecen influir en el correcto seguimiento de las guías.

No hemos detectado un exceso de prescripción de anticoagulación en pacientes con contraindicación para la misma.

\section{Centros e investigadores participantes en el estudio INCARGAL}

Investigador principal: Alfonso Castro Beiras.

Centro coordinador: ODDS, S.L. Javier Muñiz García y Alberto García Castelo.

Hospitales e investigadores participantes: Hospital Juan Canalejo, A Coruña: Servicio de Cardiología: José Ángel Rodríguez Fernández, Beatriz Bouzas Zubeldía, Ignacio Mosquera Pérez, Lorenzo Monserrat Iglesias; Servicio de Medicina Interna: Vicente Ramos Polledo, Fernando de la Iglesia Martínez, Ricardo Nicolás Miguel, Carlos Pellicer Vázquez, Hospital Arquitecto Marcide, El Ferrol: Servicio de Medicina Interna: Pascual Sesma Sánchez, Carlos González González. Hospital Clínico Universitario, Santiago de Compostela: Servicio de Medicina Interna: Vicente Lorenzo Zúñiga, María Rosario Alende Sixto, Carmen Mella Pérez, Emilio Manuel Padín Paz, lago Villamil Cajato. Hospital De Conxo, Santiago de Compostela: Servicio de Medicina Interna: Antonio Pose Reino. Hospital Xeral-Calde, Lugo: Servicio de Cardiología: Juan Vidán Martínez, Ricardo Izquierdo González; Servicio de Xeriatría: Fernando Veiga Fernández, José Ramón Martínez Calvo, Manuel Melero Brezo. Hospital Comarcal Da Costa, Burela: Servicio de Cardiología: José Antonio Lombán Villanueva. Hospital Comarcal de Monforte: Servicio de Cardiología: Nicolás Bayón Meleiro. Hospital Santa Maria Nai, Ourense: Servicio de Cardiología: Miguel Pérez de Juan Romero, M. Dolores Collell Mach, Rosa Mojón Perezy; Servicio de Medicina Interna: Manuel de Toro Santos, Amalia Cadavid Rodríguez, Serafín Pérez Pombo, Justa Rego Villa Amor, Miguel Ángel Rodríguez Quintela, Elvira Rodríguez Torres. Hospital Cristal Piñor, Ourense: Servicio de Cardiología: Evaristo Freire Castroseiros; Servicio de Medicina Interna: Ovidio Fernández, Manuel Jiménez Martínez. Hospital Comarcal de Valdeorras: Servicio de Medicina Interna: Josep Masferrer Serra, A. Eneriz Calvo. Hospital Xeral-Cíes, Vigo: Servicio de Cardiología: Hugo Torrealday Taboada, José Penas Cortés; Servicio de Medicina Interna: Bernardo Sopeña López. Hospital do Meixoeiro, Vigo: Servicio de Cardiología: M. Victoria Platero Vázquez, E. Martín, Estrella Pérez Fernández; Servicio de Medicina Interna: José Carlos Medraño Martínez; Servicio de Geriatría: Carlos Rodríguez Pascual, M. Luz López Blanco, M. Teresa Olloz Chiva. Hospital Montecelo, Pontevedra: Servicio de Cardiología: Juan Raúl Casariego Rosón, Rodrigo Medina Alba, Benito Puente Rodero, Pedro Vigil Escalera. Hospital Provincial de Pontevedra: Servicio de Cardiología: Manuel Silva Martínez, Concepción Fernández Costa, M. José Pedros Cuadrillero; Servicio de Medicina Interna: Antonio Martínez Muradas.

\section{Bibliografía}

1. Krahn AO, Manfreda J, Tate RB. Mathewson FAL, Cuddy ET. The natural history of atrial fibrillation: incidence, risk factors, and prognosis in the Mannitoba Follow-up Study. Am J Med 1995;98:476-84.

2. Garg R, Yusuf S, Pouler OB, editors. Congestive cardiac failure: pathophysiology and treatment. Epidemiology of Congestive Cardiac Failure, 1993; p. 9-25.

3. Jordaens L, Trouerbach J, Calle P, Tavernier R, Oerycke E, Vertongen P, et al. Conversión of atrial fibrillation to sinus rhythm and rate control by digoxin in comparison to placebo. Eur Heart J 1997; 18:643-8.

4. Johnstone O, Limacher M, Rousseau M, Liang CS, Ekelund L, Herman M, et al. Clinical characteristics of patients in studies of left ventricular dysfunction (SOL VD). Am J Cardiol 1992;70: 894-900.

5. Swedberg K, Idanpaan Heikkila U, Remes J, and CONSENSUS Trial Study Group. Effects of enalapril on mortality in severe congestive heart failure. Results of the Coperative North Scandinavian Enalapril Survival Study (CONSENSUS). N Engl J Med 1987;316: 1429-35. 
6. Wolf PA, Abbott RO, Kannel WB. Atrial fibrillation as an independent risk factor for stroke: the Frarningharn Study. Stroke $1991 ; 22: 983-8$.

7. Atrial Fibrillation Investigators. Risk factors for stroke and efficacy of antithrombotic therapy in atrial fibrillation. Analysis of pooled data from five randomized controlled trials. Arch Intern Med 1994;154:1449-57.

8. Morley J, Marinchak R, Rials SJ, Kowey P. Atrial fibrillation, anticoagulation, and stroke. Am J Cardiol I 996;77:A38-44.

9. Petersen P, Boysen G, Godtfredsen J, Andersen ED, Andersen B. Placebo-controlled, randomised trial of warfarin and aspirin for prevention of thromboembolic complications in chronic atrial fibrillation. The Copenhagen AFASAK Study. Lancet 1989; 1: 175-9.

10. The Boston Area Anticoagulation Trial for Atrial Fibrillation Investigators. The effect of low-dose warfarin on the risk of stroke in patients with nonrheumatic atrial fibrillation. N Engl J Med 1990;323: 1505-1 1.

11. Stroke Prevention in Atrial Fibrillation Investigators. Stroke prevention in atrial fibrillation study. Final results. Circulation 1991; 84:527-39.

12. Connolly SJ, Laupacis A, Gent M, Roberts RS, Cairns JA, Joyner C, for the CAFA Study Coinvestigators. Canadian Atrial Fibrillation Anticoagulation (CAFA) study. J Am Coll Cardiol 1991; 18: 349-55.

13. Ezekowitz MD, Bridgers SL, James KE, Carliner NH, Colling CL, Gornick CC, et al, for the Veterans Affairs Stroke Prevention in nonrheumatic Atrial Fibrillation lnvestigators. Warfarin in the prevention of stroke associated with nonrheumatic atrial fibrillation. N Engl J Med 1992;327: 1406-12.

14. EAFT (European Atrial Fibrillation Trial) Study Group. Secondary prevention in non-rheumatic atrial fibrillation after transient ischaemic attack or minor stroke. Lancet 1993;342: 1255-62.

15. Stroke Prevention in Atrial Fibrillation Investigators. Warfarin versus aspirin for prevention of thromboembolism in atrial fibrillation: Stroke Prevention in Atrial Fibrillation II Study. Lancet 1994;343:687-91.

16. Stroke Prevention in Atrial Fibrillation Investigators. Adjusted-dose warfarin versus low-intensity, fixed-dose warfarin plus aspirin for high-risk patients with atrial fibrillation: Stroke Prevention in Atrial Fibrillation III randomised clinical trial. Lancet 1996;348:633-8.

17. Khand AU, RankinAC, Kaye GC, Cleland GF. Systematic review of the management of atrial fibrillation in patients with heart failure. Eur Heart J 2000;21:614-32.

18. Almendral J, Marín E, Medina O, Peinado R, Pérez L, Ruiz R, et al. Guías de práctica clínica en arritmias cardíacas. Rev Esp Cardiol 200 1;54:307-67.

19. Heras M, Fernández A, Gómez JA, Iriarte JA, Lidón RM, Pérez F, et al. Guías de actuación clínica de la Sociedad Españo- la de Cardiología. Recomendaciones para el uso del tratamiento antitrombótico en cardiología. Rev Esp Cardiol 1999;52: 801-20.

20. Fuster V, Rydén LE, Asinger RW, Cannom DS, Crijns HJ, Frye RL, et al. ACC/AHA/ESC guidelines for the management of patients with atrial fibrillation: a report of the American College of Cardiology/American Heart Association Task Force on Practice Guidelines and the European Society of Cardiology Committee for Practice Guidelines and Policy Conferences (Committee to Develop Guidelines for the Management of Patients With Atrial Fibrillation). J Am Coll Cardiol 200 1;38: 1231-66.

21. Brotons C, Moral 1, Antón JJ, Cobos M, Cucurull E, Gállego C, et al. Tratamiento preventivo de la fibrilación auricular no reumática: de la eficacia de los ensayos clínicos a la efectividad en la práctica clínica. Atenc Primaria 1997;20:367-71.

22. Sánchez IF, García L, Chiquero M, Lozano G, Pérez S, Alonso T, et al. Tratamiento antitrombótico en la fibrilación auricular no reumática. ¿Cumplimos las recomendaciones de los ensayos clínicos? An Med Interna 1999; 16:569-73.

23. Frykrnan V, Beerman B, Rydén L, Rosenqvist M. Management of atrial fibrillation: discrepancy between guideline recommendations and actual practice ex poses patients to risk for complications. Eur Heart 1200 1;22: 1954-9.

24. García Castelo A, Muñiz García J, Sesma Sánchez P, Castro Beiras A. Utilización de recursos diagnósticos y terapéuticos en pacientes ingresados por insuficiencia cardíaca: influencia del servicio de ingreso (estudio INCARGAL). Rev Esp Cardiol 2003;56: 49-56.

25. Nademanee K, Kosar EM. Long-term antithrombotic treatment for atrial fibrillation. Am J Cardiol I 998;82:N3742.

26. Hylek EM, Singer DE. Risk factors for intracraneal hemorrhage in outpatients taking warfarin. Ann Intern Med 1994;120:897- 902 .

27. Fihn SO, Callahan CM, Martin OC, McDonell MB, Henikoff JG, White RH, for The National Consortium of Anticoagulation Clinics. The risk for and severity of bleeding complications in elderly patients treated with warfarin. Ann Intern Med 1996; 124: 970-9.

28. The Stroke Prevention in Atrial Fibrillation Investigators. Bleeding during antithrombotic therapy in patients with atrial fibrillation. Arch Intern Med 1996; 156:409-16.

29. Gullov AL, Koefoed BG, Petersen P. Bleeding during warfarin and aspirin therapy in patients with atrial fibrillation. The AFA- SAK 2 Study. Arch Intern Med 1999; 159: 1322-8.

30. Cairns JA, Connolly SJ. Nonrheumatic atrial fibrillation: risk of stroke and role of antithrombotic therapy. Circulation $1991 ; 84:$ 469-81.

31. Antiplatelet Trialists' Collaboration. Collaborative overview of randomised trials for antiplatelet therapy-I: Prevention of death, myocardial infarction, and stroke by prolonged antiplatelet therapy in various categories of patients. BMJ 1994;308:81-106. 
32. Anticoagulants in the Secondary Prevention of Events in Coronary Thrombosis (ASPECT) Research Group. Effect of long-terrn oral anticoagulant treatment on mortality and cardiovascular morbidity after myocardial infarction. Lancet 1994;343:499-503.

33. The EPSIM Research Group. A controlled comparison of aspirin and oral anticoagulants in prevention of death after myocardial infarction. N Engl J Med 1982;307:701-8.

34. Julian DG, Chamberlain DA, Pocock SJ, for the AFTER Study Group. A comparison of aspirin and anticoagulation following thrombolysis for myocardial infarction (the AFTER study): a multicentre unblinded randomised clinical trial. BMJ 1996;313: 1429- 31.

35. Fuster Y, Yerstraete M. Heart disease. A textbook of cardiovascular medicine. 5th ed. Philadelphia: Saunders, 1997; p. 1828-9.

36. Anand SS, Yusuf S. Oral anticoagulant therapy in patients with coronary artery disease: a meta-analysis. JAMA 1999;282:2058- 67.

37. Gull_v AL, Koefoed BG, Petersen P, Pedersen TS, Andersen ED, Godtfredsen 1, et al. Fixed mini-dese warfarin and aspirin alone and in combination compared with adjusted-dose warfarin for stroke prevention in atrial fibrillation. Second Copenhagen Atrial Fibrillation, Aspirin and Anticoagulation Study. Arch Intern Med 1998;158:1513-21. 